\title{
Dispositivo Não Invasivo para Monitoramento do Ambiente do Sono
}

\author{
Josué A. S. Oliveira ${ }^{1}$, Vitor S. Campos ${ }^{1}$, Josivan R. Reis ${ }^{1}$, \\ Fábio M. F. Lobato ${ }^{1}$, Roberto P. Nascimento ${ }^{1}$ \\ ${ }^{1}$ Universidade Federal do Oeste do Pará (UFOPA) - Santarém, PA - Brasil \\ \{josuefan56, vituca.9\}@gmail.com \\ \{josivan.reis, fabio.lobato, roberto.nascimento\}@ufopa.edu.br
}

\begin{abstract}
Sleep disorders are recurrent problems in society and have gained attention in recent years, especially when considering the changes imposed by Information and Communication Technologies (ICT) because they allow the development of solutions aimed at the evaluation of sleep. These disorders are usually associated with some clinical condition and usually the diagnosis is obtained through laboratory polysomnography, however, this is an expensive technique and may be inconvenient to patients. Therefore, it is worth emphasizing the importance of the sleep environment in the context of the diagnosis, since the variables associated to it play an important role in sleep quality. In this sense, the article presents the development of a non-invasive and low-cost device for monitoring the sleep environment, designed under the Design Science Research Methodology, to aid in the treatment of sleep disorders.
\end{abstract}

Resumo. Os distúrbios do sono são problemas recorrentes na sociedade e tem ganhado atenção nos últimos anos, sobretudo quando consideradas as mudanças impostas pelas Tecnologias da Informação e Comunicação (TIC) por permitirem o desenvolvimento de soluções direcionadas à avaliação do sono. Esses distúrbios geralmente estão associados à alguma condição clínica e geralmente o diagnóstico é obtido por meio da da polissonografia laboratorial, no entanto, essa é uma técnica dispendiosa e que pode ser inconveniente aos pacientes. Diante disso, vale destacar a importância do ambiente do sono no contexto do diagnóstico, já que as variáveis associadas a ele desempenham um importante papel na qualidade do sono. Nesse sentido, apresenta-se neste artigo, o desenvolvimento de um dispositivo não invasivo e de baixo custo para monitoramento do ambiente de sono, concebido sob o Design Science Research Methodology, com objetivo de auxiliar no tratamento dos distúrbios do sono.

\section{Introdução}

O sono é um processo ativo do cérebro humano que tem a função biológica fundamental na consolidação da memória, na visão binocular, na termorregulação, na conservação e restauração da energia [Jansen et al. 2007]. Porém, essa função reparadora do sono pode ser comprometida se a pessoa sofre com perturbações ou distúrbios durante o sono, já que isso pode acarretar alterações significativas no funcionamento físico, ocupacional, cognitivo e social do indivíduo, além de comprometer substancialmente a qualidade de vida [Müller and Guimarães 2007]. Segundo dados da Organização Mundial da Saúde, cerca 
de $40 \%$ da população mundial sofre com problemas relacionados ao sono, que muitas vezes estão correlacionados à doenças como obesidade, diabetes e doença cardiovascular, o que caracteriza um cenário preocupante de saúde pública [Buxton and Marcelli 2010].

Atualmente existem diversas técnicas para detectar se uma determinada pessoa sofre com distúrbios no sono, a mais utilizada delas é a de polissonografia [de Menezes Duarte et al. 2010]. No entanto, essa técnica requer múltiplos sensores conectados ao corpo do paciente, e também, geralmente, o deslocamento do paciente até uma clínica médica [Bittencourt and Caixeta 2010]. Essa abordagem invasiva pode afetar o resultado final do processo de monitoramento, já que o paciente pode sofrer incômodo resultante dos fios dos sensores, além de não se encontrar em seu ambiente normal de sono. O monitoramento, análise e diagnóstico são obtidos através do registro simultâneo de alguns parâmetros fisiológicos do sono do paciente [Guimarães 2010].

Sistemas de monitoramento baseados em microcontroladores têm sido amplamente utilizados em muitos domínios de aplicação. Entre os projetos existentes para o monitoramento do sono, pode-se mencionar: o sistema de monitoramento baseado no sensor do Kinect para coletar dados do movimento, postura e do ambiente e a partir desses dados extrair informações gerais sobre o sono [Lee et al. 2015]. Sistemas de reconhecimento contínuo do estado do sono, incluindo os movimento e as saídas do usuário da cama e o monitoramento da respiração do usuário já foram descritos na literatura [Lobato et al. 2015, Lin et al. 2016, Lobato et al. 2017]. Já o [Nam et al. 2016] desenvolveu um sistema de monitoramento para quantificar a qualidade do sono.

Com base na análise das variáveis do ambiente de sono do paciente (luminosidade, ruído, movimento, distância, temperatura e umidade) pretende-se prover informações mais acuradas sobre o descanso dos pacientes sob escrutínio. Desenvolvido sob a ótica do Design Science Research (DSR), o artefato possui como principal diferencial a avaliação da qualidade do sono in loco, não sendo necessário retirar o paciente de sua residência, reduzindo custos.

Nesse sentido, a utilização de uma técnica não invasiva é mais interessante e adequada ao contexto de monitoramento do paciente. Sob essa justificativa, o presente artigo, descreve o desenvolvimento de um dispositivo para monitoramento não invasivo do ambiente do sono, que realiza a coleta, armazenamento e análise das variáveis ambiente. Em estágios finais do processo de monitoramento, os dados devem estar modelados e correlacionados, sendo possível observar as combinações resultantes dessas variáveis, de forma que indiquem alguma anormalidade no sono do paciente e que permita o diagnóstico. $\mathrm{O}$ dispositivo está sendo projetado de forma que o monitoramento possa ser feito remotamente pelo médico e a equipe técnica.

Além desta introdução, o artigo contempla mais 4 seções. Na seção 2 é definida a metodologia e os materiais utilizado no desenvolvimento do sistema. Já na seção 3 estão descritas em módulos, o desenvolvimento do protótipo. Na seção 4 é descrito o funcionamento do artefato. Por fim, na seção 5 são apresentados os resultados e discussões.

\section{Materiais e Métodos}

Para o desenvolvimento deste trabalho adotou-se a metodologia de pesquisa Design Science, tendo em vista que essa fornece uma base para conduzir uma pesquisa sólida e 
relevante, uma vez que, pretende-se desenvolver um dispositivo que auxilie na avaliação da qualidade do sono, medindo as variáveis ambientes presentes no local de repouso do paciente. O motivo para a utilização dessa metodologia é que ela permite uma rápida prototipação do produto.

O protótipo foi construído baseado no kit de desenvolvimento JETSON Tegra K1. A placa conta com baixo consumo elétrico e alto poder de processamento em relação a outros sistemas embarcados, além de contar com uma GPU (Graphics Processing Unit) para realizar o processamento de dados complexos, segundo o site oficial da Nvidia ${ }^{1}$, a placa conta com uma CPU ARM Cortex -57, com quatro núcleos de processamento, dessa forma, o torna ideal para implementar o terceiro módulo de processamento e análise de dados coletados do dispositivo.

A conexão dos sensores foi feito usando os protocolos de comunicação GPIO (General Purpose Input Output) e I2C através das portas de expansão presentes na placa, porém, como a maioria dos sensores presentes no mercado trabalham na faixa de $3,3 \mathrm{~V}$ a $5 \mathrm{~V}$, foi necessário utilizar um comutador lógico bidirecional para diminuir a voltagem do sinal recebido, pois as portas de expansão da JETSON TK1 trabalham em no máximo $1,8 \mathrm{~V}$.

O projeto conta com a implementação de cinco sensores, que têm como função realizar o monitoramento do ambiente do sono do paciente. Os sensores utilizados foram: sensor de presença (PIR DYP MR003), sensor de som (KY-038), sensor ultrassônico (HCSR04), sensor de luminosidade (BH1750) e o sensor de temperatura e umidade (AM2302 - DHT22).

O banco de dados utilizado para armanezar as leituras realizadas in loco, foi o SQlite ${ }^{2}$, por ser simples e trabalhar de forma embutida, adequado ao contexto de desenvolvimento do dispositivo.

\section{Desenvolvimento do Protótipo}

Após definidos os requisitos básicos do dispositivo iniciou-se a fase de desenvolvimento. Em um visão geral, o dispositivo multimodal terá o seu funcionamento baseado em 3 módulos interdependentes, que pode ser observado na Figura 1, sendo o módulo (1) aquisição dos dados do ambiente, que realiza a coleta dos dados por meio dos sensores, (2) diário do sono, que visa manter informações relevantes sobre o paciente e (3) análise e relatório, que trata da análise dos dados e gera os relatórios para equipe técnica.

Para o primeiro módulo buscou-se sensores com valores acessíveis no mercado e um kit de desenvolvimento integrado. A implementação dos sensores utilizou a linguagem de programção ANSI C++ em conjunto com um banco de dados SQLite.

O segundo módulo contém a interface do sistema, construída em Python e que utiliza a biblioteca Flask ${ }^{3}$ que possui um modelo simples para desenvolvimento web. Esse módulo é o responsável por permitir o acesso ao sistema por meio dos diversos dispositivos móveis, como por exemplo: computador, smartphone, tablet etc. Os questionários são implementados utilizando perguntas referentes as condições relevantes sobre o sono.

\footnotetext{
${ }^{1}$ https:/www.nvidia.com.br/

${ }^{2} \mathrm{https} / / / \mathrm{www}$. sqlite.org/

${ }^{3}$ http://flask.pocoo.org/
} 


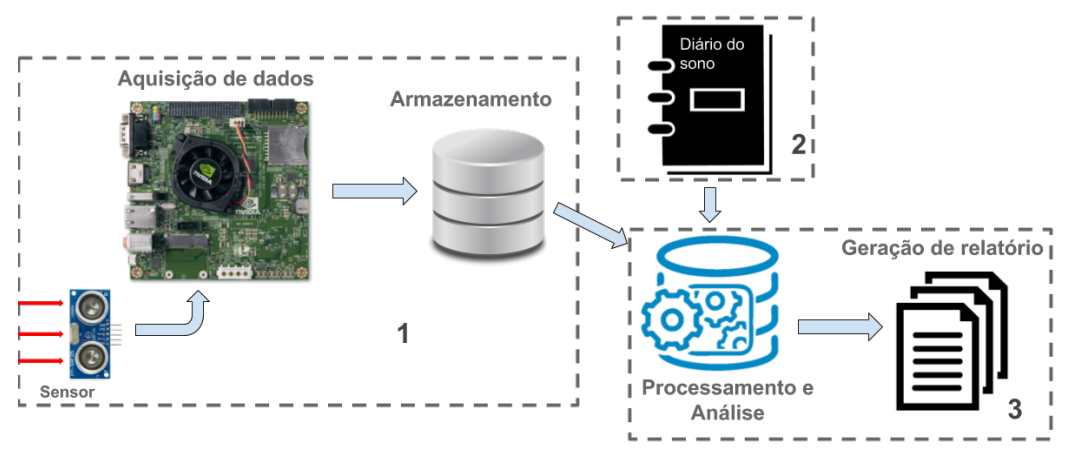

Figura 1. Esquema de funcionamento dos módulos.

Aqui também utiliza-se o banco de dados SQLite, cujo diagrama do modelo relacional está ilustrado na Figura 2.

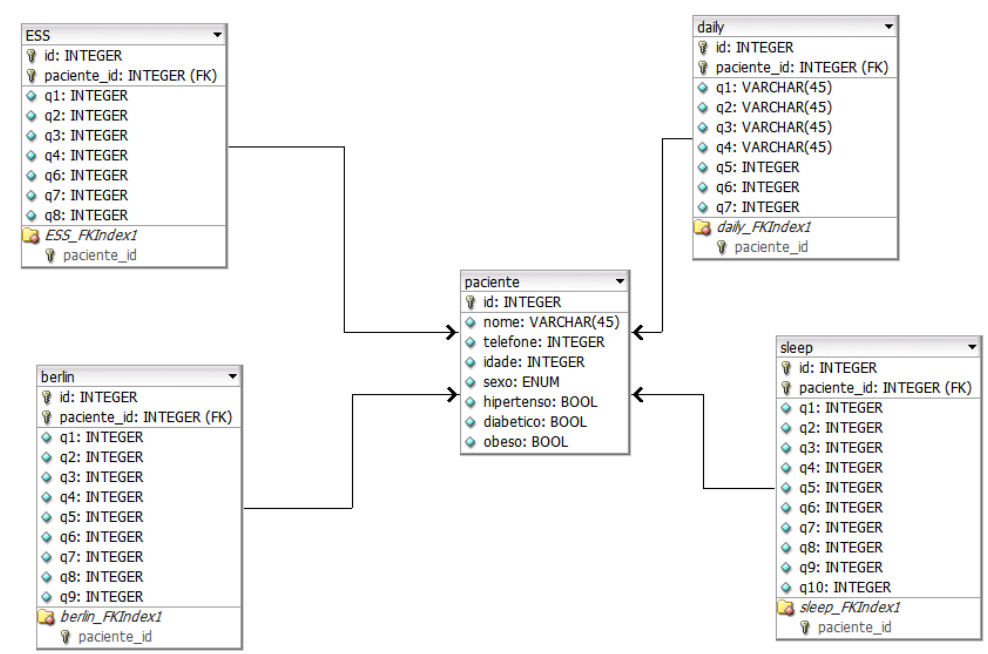

Figura 2. Diagrama do modelo relacional do banco de dados dos questionários.

Por fim, tem-se o módulo de análise e relatório, que de provê informações aos médicos e pessoal técnico envolvido no diagnóstico e tratamento dos distúrbios do sono. Com a obtenção de informações sobre eventos que ocorrem durante o sono, esses dados são correlacionados aos questionários obtidos produzindo um resumo dos eventos ocorridos, tais como, a quantidade de vezes que o paciente levantou da cama, o número de movimentos, os níveis de ruído (em função do tempo) etc. Nesse sentido, o diagnóstico de um paciente, a considerar as múltiplas variáveis que condicionam o sono normal, pode mostrar-se relativamente complexo.

\section{Funcionamento do dispositivo}

O dispositivo é orientado à eventos, o que significa que um ruído com um elevado grau de intensidade ou uma variação brusca de temperatura compõe um evento. Por exemplo, quando um dos sensores detecta presença de uma pessoa no ambiente ou baixa luminosidade (indicando possivelmente a luz do ambiente desligada) os outros sensores são acionados, como o de temperatura e umidade, som e distância para o monitoramento das variáveis do ambiente. 
Dessa forma, esses dados são armazenados em uma base de dados para posterior processamento e análise, em conjunto com os questionários do sono. Esses questionários são preenchidos por meio de uma página web que contém os dados cadastrais do referido paciente. A autenticação no sistema é baseada em login e senha. Todos os dados obtidos serão utilizados para compor médias e gráficos de forma que as informações mais relevantes estejam presentes no relatório técnico, já que períodos de monitoramento em que não é observável alterações significativas nas variáveis do ambiente, serão consideradas apenas para efeito de análise.

O sistema deve fornecer robustez, escalabilidade e praticidade para monitoramento do ambiente do sono, e ainda contar com a facilidade de fazê-lo in loco e possuir autonomicidade, sem a necessidade de operação com equipamentos de caráter complexo, que configura cenários de desconforto ao paciente e que normalmente possuem alto custo de aquisição.

\section{Resultados e Discussões}

Testes foram realizados com o dispositivo, de modo que o funcionamento dos módulos implementados do sistema de monitoramento foi verificado, assim como a inserção e busca de informação no banco de dados. Além disso, os sensores e demais periféricos estão sendo testados.

O primeiro módulo, que trata dos sensores, está parcialmente implementado, com três sensores funcionando corretamente dos cinco pretendidos. Os sensores de ruído, presença e ultrassônico estão coletando os dados e já armazenando no banco de dados, enquanto que os de luminosidade e de temperatura e umidade necessitam de mais testes. A Figura 3(a) apresenta o protótipo com os sensores implementados até o momento.

O segundo módulo já está concluído e conta com os questionários, interface web e diários do sono implementados. A Figura 3(b) mostra um exemplo de questionário utilizado no sistema. Já o terceiro módulo que trata da análise e relatório dos dados encontra-se em vias de implementação.

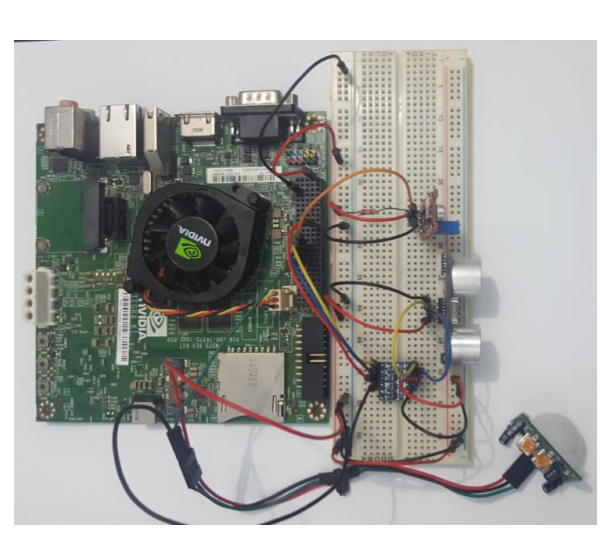

(a) Protótipo preliminar.

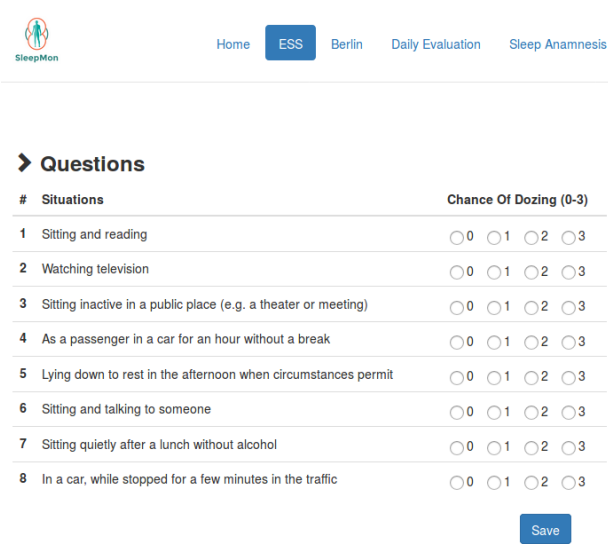

(b) Modelo de questionário utilizado para obter dados relativos ao sono do paciente.

Figura 3. Sistema Proposto.

O módulo de análise e relatório ainda está em fase de implementação mostrando- 
se promissor, cujo principal obstáculo é a ausência de heurísticas corretas que correlacionem os dados obtidos tendo em vista o diagnóstico.

\section{Agradecimentos}

Os autores gostariam de agradecer à FAPESPA pelas bolsas de iniciação científica concedidas. E também à NVIDIA pela doação do Jetson Development Kit Tk1 utilizadas nos experimentos.

\section{Referências}

Bittencourt, L. R. A. and Caixeta, E. C. (2010). Critérios diagnósticos e tratamento dos distúrbios respiratórios do sono: Saos. Jornal Brasileiro de Pneumologia.

Buxton, O. M. and Marcelli, E. (2010). Short and long sleep are positively associated with obesity, diabetes, hypertension, and cardiovascular disease among adults in the united states. Social science \& medicine, 71(5):1027-1036.

de Menezes Duarte, R. L., da Silva, R. Z. M., and da Silveira, F. J. M. (2010). Métodos resumidos no diagnóstico da apnéia do sono. Pulmão RJ, 19(3-4):78-82.

Guimarães, G. M. (2010). Diagnóstico polissonográfico. Pulmão.(Rio de Janeiro), 9(34):88-92.

Jansen, J. M., Lopes, A. J., Jansen, U., Capone, D., Maeda, T. Y., Noronha, A., and Magalhães, G. (2007). Medicina da noite: da cronobiologia à prática clínica. SciELOEditora FIOCRUZ.

Lee, J., Hong, M., and Ryu, S. (2015). Sleep monitoring system using kinect sensor. International Journal of Distributed Sensor Networks, 11(10):875371.

Lin, F., Zhuang, Y., Song, C., Wang, A., Li, Y., Gu, C., Li, C., and Xu, W. (2016). SleepSense: A Noncontact and Cost-Effective Sleep Monitoring System. IEEE Transactions on Biomedical Circuits and Systems, 11(1):189-202.

Lobato, F., Silva, B., Bem, R., and Miranda, D. (2015). Non-invasive sleep-environment monitoring system. In PETRA '15: Proceedings of the 8th International Conference on PErvasive Technologies Related to Assistive Environments, Corfu, Greece. ACM.

Lobato, F. M. F., de Resende, D. C. O., do Nascimento, R. P., Siqueira, A. L. C., Jacob, A. F. L., and de Santana, Á. L. (2017). Multimodal Low-Invasive System for Sleep Quality Monitoring and Improvement. In Batalla, J. M., Mastorakis, G., Mavromoustakis, C. X., and Pallis, E., editors, Beyond the Internet of Things: Everything Interconnected, pages 223-242. Springer International Publishing, Cham.

Müller, M. R. and Guimarães, S. S. (2007). Impacto dos transtornos do sono sobre o funcionamento diário e a qualidade de vida. Estudos de psicologia, 24(4):519-528.

Nam, Y., Kim, Y., and Lee, J. (2016). Sleep monitoring based on a tri-axial accelerometer and a pressure sensor. Sensors, 16(5):1-14. 